Article

\title{
Atomistic Investigation of Anisotropic Nanoindentation Behavior of Nanotwinned Aluminum Containing Inclined Twin Boundaries
}

\author{
Yuan Liu ${ }^{1}$, Yanfeng Duan ${ }^{2}$ and Junjie Zhang ${ }^{3, *}$ \\ 1 School of Astronautics, Harbin Institute of Technology, Harbin 150001, China; liuyuan_hit@hit.edu.cn \\ 2 Research Department of Structure Design and Simulation, Graduate School of Hubei Aerospace Technology \\ Academe, Wuhan 430040, China; duanyanfeng1986@163.com \\ 3 Center for Precision Engineering, Harbin Institute of Technology, Harbin 150001, China \\ * Correspondence: zhjj505@gmail.com
}

Received: 12 August 2018; Accepted: 5 September 2018; Published: 6 September 2018

check for updates

\begin{abstract}
Nanotwinned metals exhibit superior mechanical properties due to unique dislocation-twin boundary interactions. In the present work, we elucidate the microscopic deformation mechanisms and their correlations with the macroscopic mechanical response of nanotwinned Al containing inclined twin boundaries under nanoindentation by means of molecular dynamics simulations. The effect of twin boundary orientation with respect to the indented surface on the nanoindentation is evaluated. Simulation results reveal that dislocation slip, dislocation-twin boundary interaction, and twin boundary migration operate in parallel in the plastic deformation of nanotwinned Al. The inclination angle of twin boundaries with respect to indented surface has a strong influence on the interaction between individual deformation modes, which in turn leads to the anisotropic indentation behavior of nanotwinned $\mathrm{Al}$.
\end{abstract}

Keywords: aluminum; nanoindentation; twin boundary; inclination angle; molecular dynamics

\section{Introduction}

Aluminum is one of the most widely used key materials for aerospace applications due to its low density, high strength, high wear resistance, and good workability. In particular, mechanical properties play a critical role in determining the performance of aluminum-based components and devices. The nanoindentation technique has been widely used to derive intrinsic mechanical properties, such as Young's modulus and hardness, of different types of materials at the micro/nanoscale [1,2].

With the development of hierarchically structured materials, interface engineering has been playing an important role in tailoring mechanical properties of materials. For instance, nanotwinned metals containing imbedded twin boundaries (TBs) show extraordinary properties of high electrical conductivity, high strength, and considerable ductility. While strengthening originates from TB blocking dislocation motion, ductility results from TB acting as a dislocation source or from TB migration itself [3-6]. For aluminum in particular, Bufford et al. reported a high work-hardening capacity and plasticity in twinned $\mathrm{Al}$ [7].

The alignment of TBs plays a central role in determining the mechanical behavior of twinned metals. Wei et al. found that the plasticity of twinned metallic nanowires can be dominated by TB-associated mechanisms by designing the inclination angle of TBs [8]. Stukowski et al. showed that the dislocation-TB intersection in nanotwinned $\mathrm{Cu}$ under uniaxial tension can be altered by the TBs' orientation with respect to the loading direction [9]. Brown et al. showed that the plasticity in twinned Cu nanopillars greatly depends on the TBs' orientation with respect to the loading axis [10]. Zhang et al. 
investigated the effect of the inclination angle on the nanoscratching behavior of nanotwinned copper, and found a critical inclination angle of $26.6^{\circ}$ for the lowest yield strength and the highest friction coefficient [11]. However, there is rather limited work focusing on the effect of the inclination angle of TBs on the mechanical properties of aluminum.

Therefore, in the present work, we perform molecular dynamics (MD) simulations to investigate the deformation mechanisms of nanotwinned Al containing inclined TBs under nanoindentation. The microscopic deformation mechanisms of materials are elucidated and are correlated with the macroscopic response of nanoindentation. We further investigate the effect of the inclination angle of TBs with respect to the indented surface on the nanoindentation process.

\section{Simulation Method}

Figure 1 shows the atomic structures of nanotwinned $\mathrm{Al}$ specimens containing differently inclined TBs. Five inclination angles are considered, as $0^{\circ}, 26^{\circ}, 45^{\circ}, 64^{\circ}$, and $90^{\circ}$, respectively. To construct the TBs with different inclination angles, the twinned specimen containing TBs with an inclination angle of $0^{\circ}$ is first constructed by periodically changing the atomic arrangement of a single crystalline specimen along the (111) direction. Then, the refereed specimen is rotated with a 2D rotation matrix along the (110) direction. Each specimen has a dimension of $35 \mathrm{~nm}$ in length, $12 \mathrm{~nm}$ in height, and $35 \mathrm{~nm}$ in width, respectively. The $\mathrm{Al}-\mathrm{Al}$ atomic interactions within the aluminum specimen are described using an embedded atom method (EAM) [12]. The intrinsic stacking fault energy of Al predicted by the utilized EAM potential is $146 \mathrm{~mJ} / \mathrm{m}^{2}$, which agrees well with the experimental values of $120-144 \mathrm{~mJ} / \mathrm{m}^{2}$ [13].

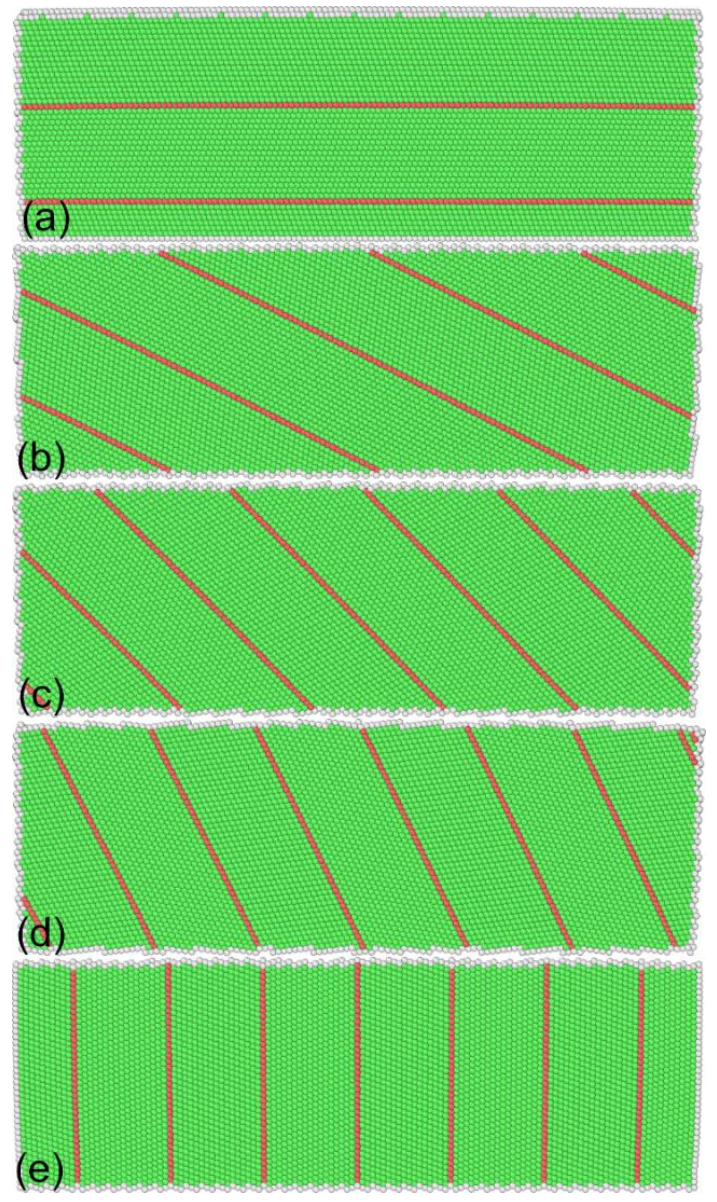

Figure 1. Atomic structures of nanotwinned $\mathrm{Al}$ containing differently inclined twin boundaries (TBs). Inclined angle of TBs: (a) $0^{\circ}$; (b) $26^{\circ}$; (c) $45^{\circ}$; (d) $64^{\circ}$; and (e) $90^{\circ}$. Atoms are colored according to their common neighbor analysis (CNA) values. 
Prior to nanoindentation, the as-constructed specimen is subjected to relaxation through the following procedures: First conjugate-gradient energy minimization at $0 \mathrm{~K}$, and then dynamic relaxation at $3 \mathrm{~K}$ and under 0 bar for 50 ps using the canonical (constant number of atoms, constant volume and constant temperature, NVT) ensemble. After reaching the equilibrium configuration, the specimen is subjected to nanoindentation using a spherical indenter. The indenter with a radius of $4 \mathrm{~nm}$ is modeled by a strong repulsive potential [14]. Nanoindentation is performed in a displacement-controlled mode, that is, the indenter penetrates the specimen surface with a constant velocity of $20 \mathrm{~m} / \mathrm{s}$ until it reaches a predetermined indentation depth of $2 \mathrm{~nm}$. The common neighbor analysis (CNA) is utilized to distinguish types of lattice defects [15]. The coloring scheme is as follows: Green stands for face-centered cubic (FCC) atoms, red for hexagonal closed packed (HCP) atoms, and gray for free surface and dislocation cores. All the MD simulations are performed using the Large-scale Atomic/Molecular Massively Parallel Simulator (LAMMPS) code with an integration step of $1 \mathrm{fs}$ [16]. Ovito is utilized to visualize MD data and generate MD snapshots [17].

\section{Results and Discussion}

\subsection{Deformation Mechanisms}

To obtain the fundamental deformation mechanisms of twinned Al, MD simulation of nanoindentation on the twinned $\mathrm{Al}$ with an inclination angle of $0^{\circ}$ is first performed. Figure 2 plots the force-displacement curve obtained, which shows that the nanoindentation can be categorized into two stages, as elastic deformation and plastic deformation, respectively. Specifically, Figure 2 shows that the transition from elastic stage to plastic stage is accompanied with a rapid decrease of indentation force, which is corresponding to the pop-in phenomenon observed in the load-controlled nanoindentation tests.

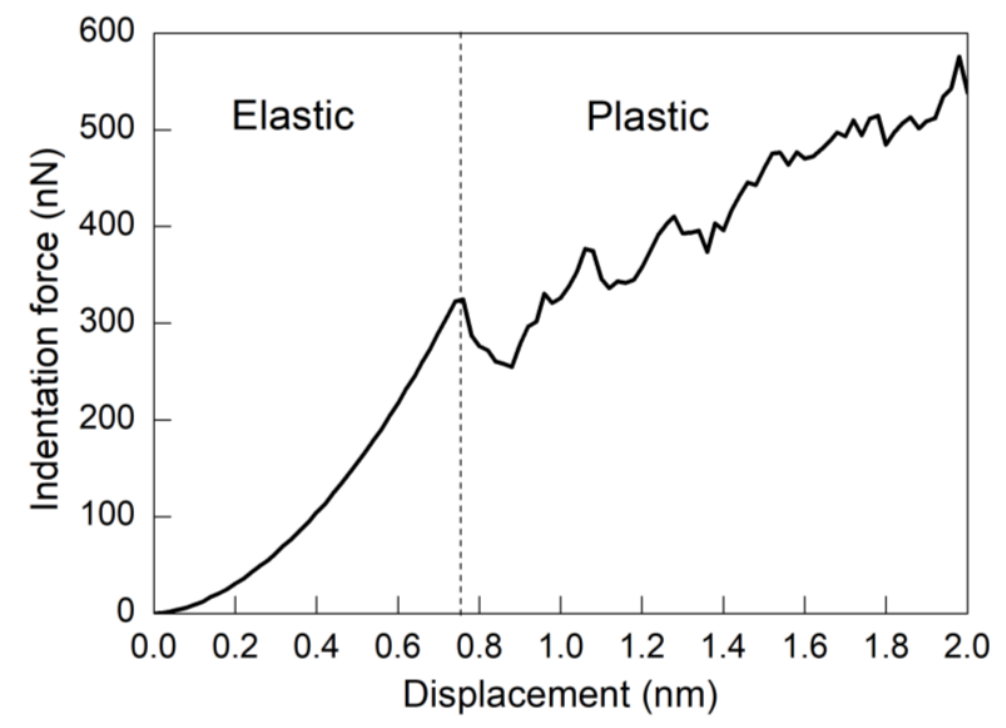

Figure 2. Force-displacement curve of twinned $\mathrm{Al}$ with an inclination angle of $0^{\circ}$ under nanoindentation.

Figure 3 presents MD snapshots of the twinned $\mathrm{Al}$ with an inclination angle of $0^{\circ}$ at different displacements. It is seen from Figure 2 that there is a rapid increase of indentation force in the elastic stage. Figure 3 a shows that, at a displacement of $0.72 \mathrm{~nm}$, the free surface in contact with the indenter is curved, but there is no internal defect generated, indicating that the material is undergoing pure elastic deformation. Upon further advancement of the indenter, a dynamic inspection of deformation behavior shows that the force drop shown in Figure 2 is caused by the emission of dislocations from surface in contact with the indenter. After nucleation, dislocation glides on the (111) slip planes along the (110) slip directions. The avalanche of dislocations releases the strain energy accumulated in the elastic stage, which leads to the force drop. However, the subsequent sliding of dislocations is 
hindered by the TBs. Figure $3 \mathrm{~b}$ shows that, at a displacement of $0.88 \mathrm{~nm}$, there is an intersection of lattice dislocations with the horizontal TB. Consequently, the TB is divided into three sections that are connected by twinning partial dislocations, that is, TB migration occurs.
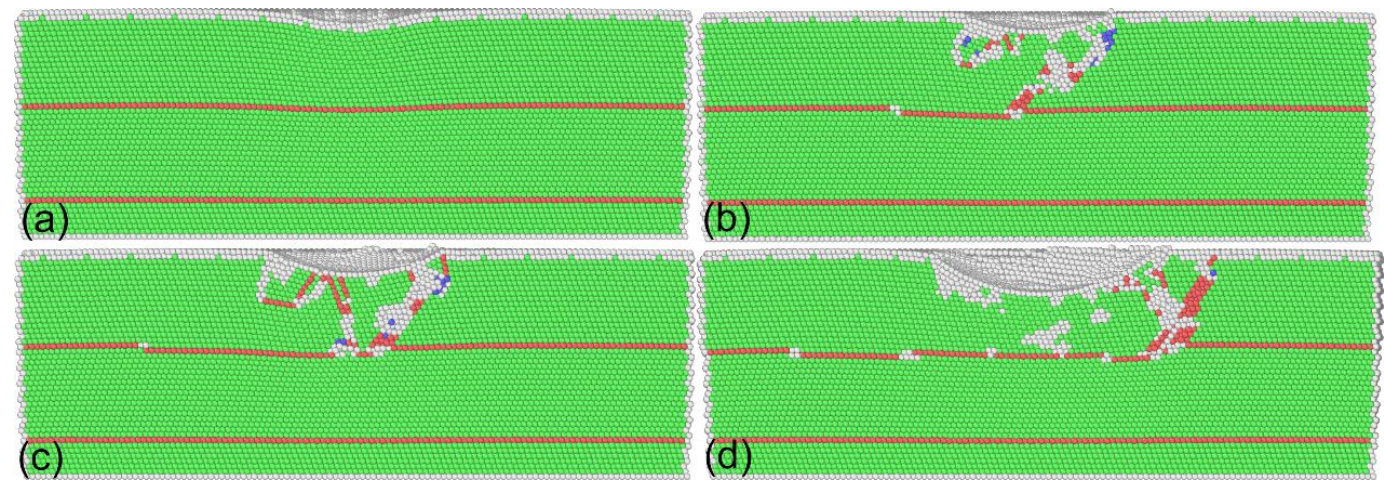

Figure 3. Cross-sectional views of twinned $\mathrm{Al}$ with an inclination angle of $0^{\circ}$ under different displacements. Displacement: (a) $0.72 \mathrm{~nm}$; (b) $0.88 \mathrm{~nm}$; (c) $1.12 \mathrm{~nm}$; and (d) $2.0 \mathrm{~nm}$. Atoms are colored according to their CNA values.

Figure $4 \mathrm{a}$ further presents the instantaneous zoom view of the dislocation-TB interaction at a displacement of $0.88 \mathrm{~nm}$. It is found from Figure $4 \mathrm{a}$ that only a portion of TB is migrated to the distance of an atomic layer, and the dislocations are confined within the twin lamellae. The confinement of dislocations by TBs leads to work hardening of the indented material, which is accompanied by the increase of indentation force. Upon further indentation, fresh dislocations are successively nucleated to accommodate the plastic strain induced by the advancement of the indenter. The fluctuation events shown in the force-displacement curve are associated with the successive nucleation events of dislocations. However, dislocation motions are still confined to the first twin lamellae. Furthermore, there are more pronounced interactions between dislocation and TB, as shown in Figure 3c. Figure $4 \mathrm{~b}$ presents the instantaneous zoom view of the dislocation-TB interaction at a displacement of $1.6 \mathrm{~nm}$. While considerable dislocations in the twin lamellae exist, dislocations are mainly inclined to the TB that is parallel to the free surface. Consequently, multiple TB migrations occur. Figure $3 \mathrm{~d}$ shows that after the completion of nanoindentation, dislocation density is significantly decreased due to the absorption of dislocations by TBs, and dislocations are completely confined within the twin lamellae. Figure $3 \mathrm{~d}$ also demonstrates that the migrated TB segments are not recovered. The above results indicate that the plastic deformation of twinned Al under nanoindentation is governed by dislocation slip, dislocation-TB interaction, and TB migration in parallel. A previous study indicated that the mechanical response and dislocation motion in bicrystal Al containing TBs under nanoimprinting are not strongly influenced by the presence of TBs [18].
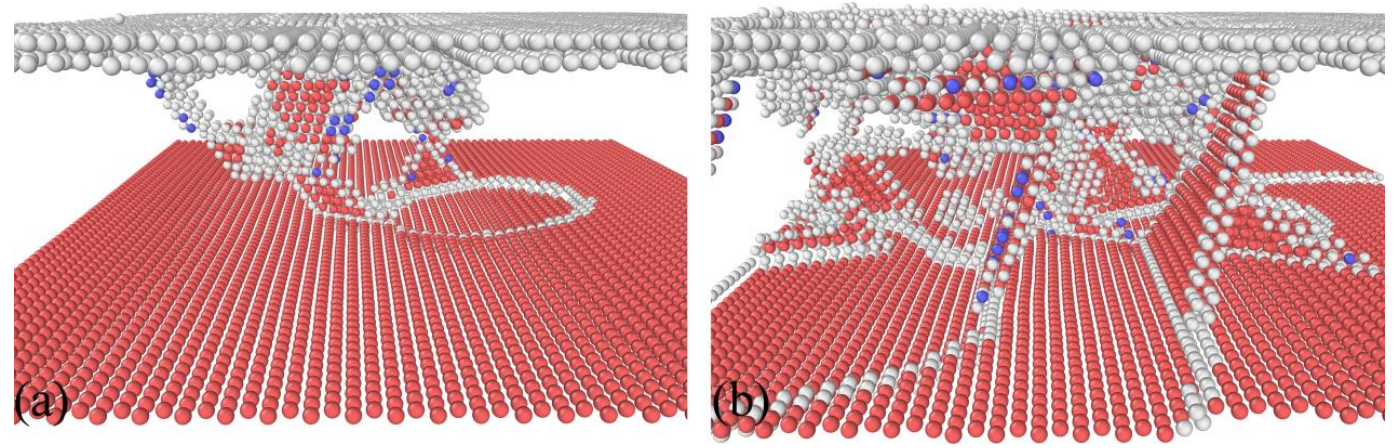

Figure 4. Representative dislocation-TB interactions. Displacement: (a) $0.88 \mathrm{~nm}$; (b) $1.6 \mathrm{~nm}$. Atoms are colored according to their CNA values, and perfect face-centered cubic (FCC) atoms are not shown. 


\subsection{Influence of Inclination Angles}

MD simulations of nanoindentation on twinned Al with differently inclined TBs are also performed to investigate the influence of the inclination angle on the nanoindentation response. Figure 5 plots the force-displacement curves during nanoindentation for different inclination angles. It is seen that for each inclination angle, the variation of indentation force has similar characteristics: It first increases rapidly in the elastic deformation, then drops at the elastic-plastic transition, and then finally increases with fluctuations in the plastic deformation. We note that the force drop phenomenon is not equally pronounced for different inclination angles, due to intrinsically different crystallographic orientations. However, the force-displacement curves for the inclination angle of $0^{\circ}$ show a higher slope than those for the other four angles, indicating a higher Young's modulus of the twinned $\mathrm{Al}$ with an inclination angle of $0^{\circ}$. For the other four inclination angles, although the force-displacement curves in the elastic deformation have approximately the same slopes, the critical displacement and associated critical force for the elastic-plastic transition are different for different inclination angles, as demonstrated in Table 1. Specifically, the inclination angle of $45^{\circ}$ has the highest critical displacement and critical force, followed by $26^{\circ}, 64^{\circ}, 0^{\circ}$, and $90^{\circ}$. After yielding, the difference in the force-displacement curves for different inclination angles is reduced due to complex deformation behavior.

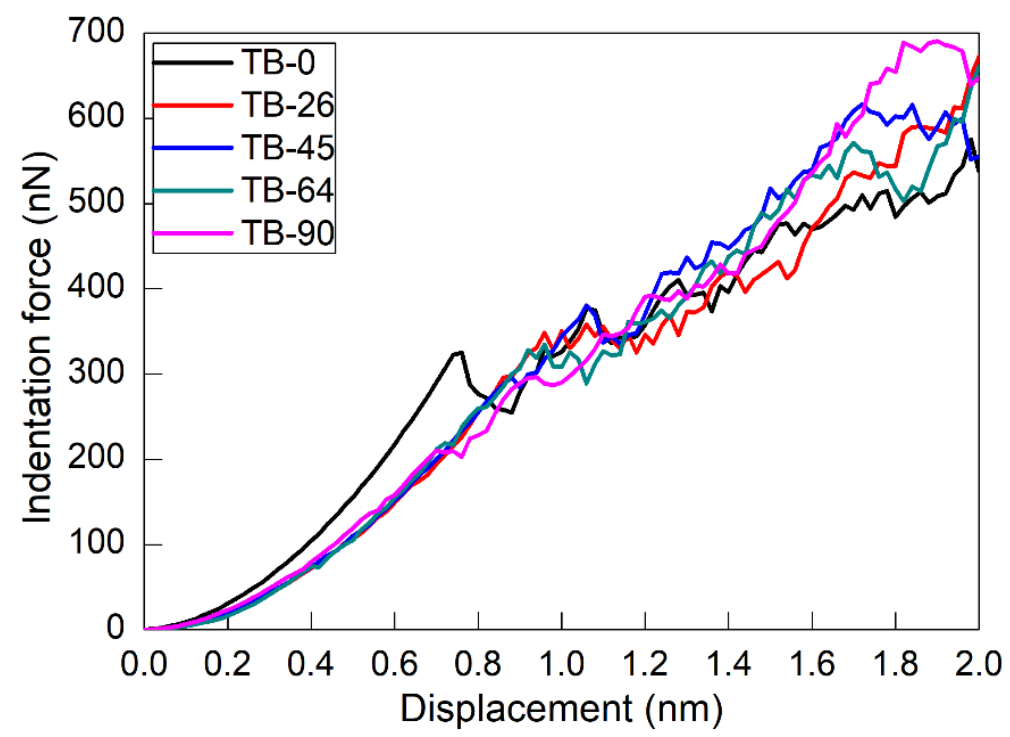

Figure 5. Force-displacement curves of twinned Al containing TBs with different inclination angles under nanoindentation.

Table 1. Critical force and displacement for different inclination angles.

\begin{tabular}{ccc}
\hline Inclination Angle & Critical Force (nN) & Critical Displacement (nm) \\
\hline $0^{\circ}$ & 326.5 & 0.76 \\
$26^{\circ}$ & 348.3 & 0.96 \\
$45^{\circ}$ & 380.8 & 1.06 \\
$64^{\circ}$ & 327.1 & 0.91 \\
$90^{\circ}$ & 209.0 & 0.70 \\
\hline
\end{tabular}

Figure 6 presents the cross-sectional views of twinned $\mathrm{Al}$ with different inclination angles after nanoindentation. Figure 7 further presents defect structures of twinned $\mathrm{Al}$ with different inclination angles after nanoindentation. As with the inclination angle of $0^{\circ}$, TBs act as barriers for dislocation motions, which also leads to TB migrations. However, the confinement of dislocation within twin lamellas is different for different inclination angles. For the inclination angle of $90^{\circ}$, there are lattice dislocations gliding parallel to TBs, in addition to inclined dislocations. However, only inclined 
dislocations are observed for the other inclination angles. Consequently, TB migration is more pronounced for the inclination angle of $90^{\circ}$ than for the other four inclination angles. For the inclination angles except for $0^{\circ}$ in particular, there are lattice dislocations emitting from the migrated position of TBs, indicating that TB acts as a dislocation source, which means dislocation activity is easier to be triggered. This also indicates the lower strength of twinned Al with an inclination angle different from $90^{\circ}$. The above results also indicate that the mean work hardening rate in the plastic regime increases with increasing inclination angle of TBs.
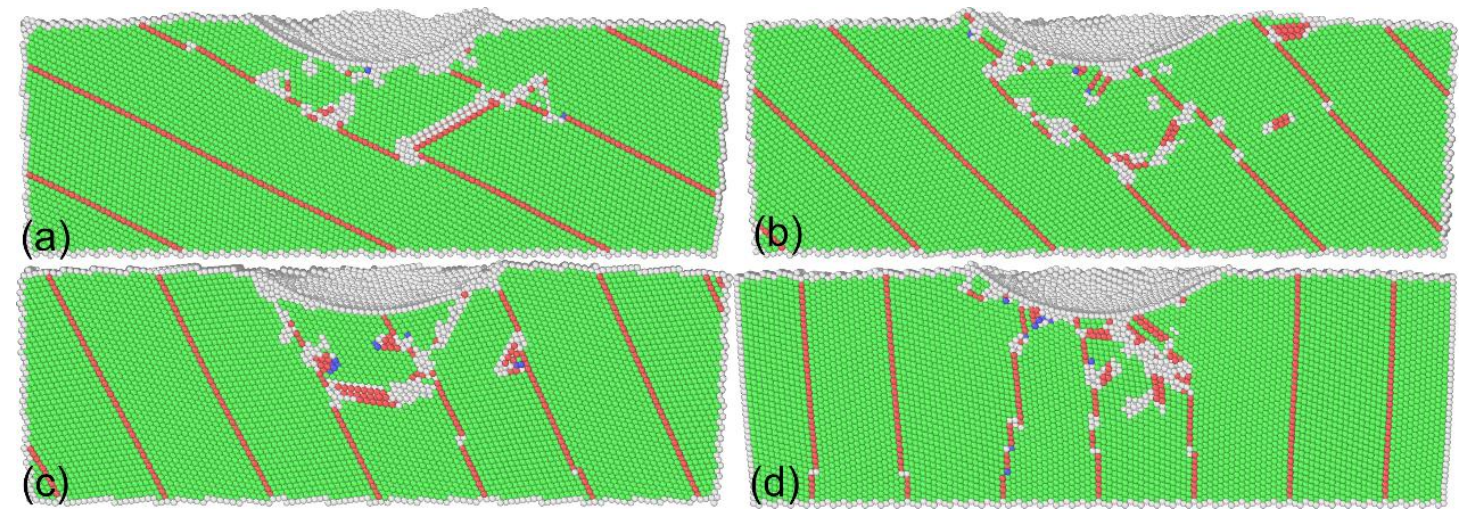

Figure 6. Cross-sectional views of twinned $\mathrm{Al}$ with different inclination angles after nanoindentation. Inclination angle: (a) $26^{\circ}$; (b) $45^{\circ}$; (c) $64^{\circ}$; and (d) $90^{\circ}$. Atoms are colored according to their CNA values.
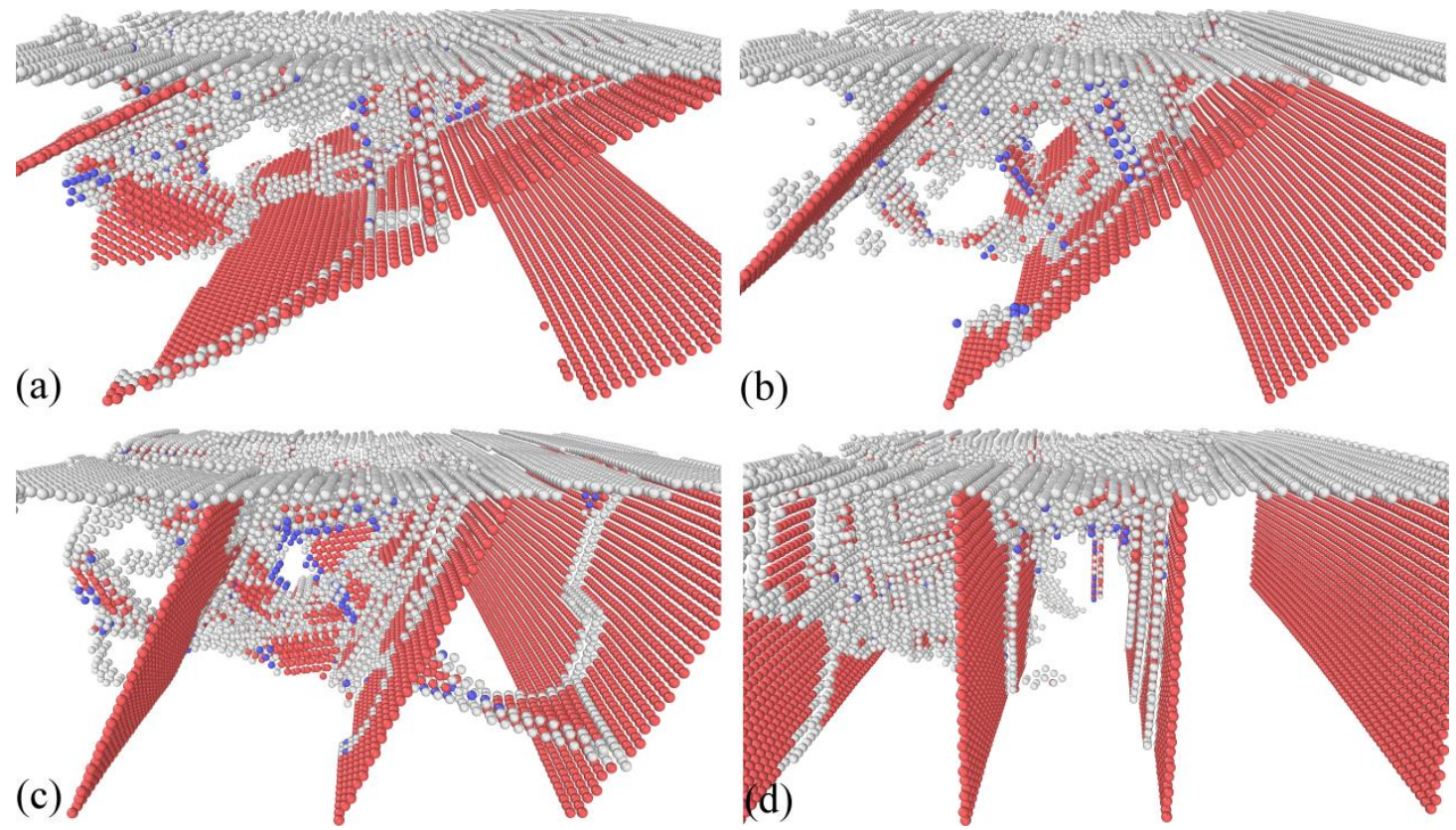

Figure 7. Representative dislocation-TB interactions. Inclination angle: (a) $26^{\circ}$; (b) $45^{\circ}$; (c) $64^{\circ}$; and (d) $90^{\circ}$. Atoms are colored according to their CNA values, and perfect FCC atoms are not shown.

Figure 8 presents the surface morphology of the indented twinned $\mathrm{Al}$ after the completion of nanoindentation. It is seen that the surface pile-up around the residual impression is significantly different for different inclination angles. The surface pile-up is most pronounced for the inclination angle of $45^{\circ}$, followed by $26^{\circ}, 64^{\circ}, 0^{\circ}$, and $90^{\circ}$. It is indicated that the surface pile-up behavior is closely associated with the strength of the twinned material, which is determined by the alignment of TBs. The propagation direction of surface pile-up is also different for different twinned materials. While there are intersections of TBs with free surface for the inclination angle of $26^{\circ}$, 
$45^{\circ}, 64^{\circ}$, and $90^{\circ}$, the propagation of displaced materials is mainly along the intersection direction. However, surface pile-up for the inclination angle of $0^{\circ}$ is different.
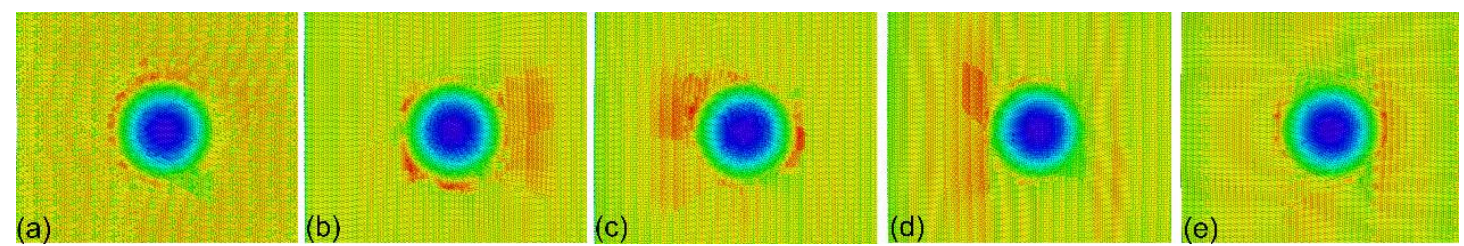

Figure 8. Surface morphology of twinned $\mathrm{Al}$ with different inclination angles. Inclination angle: $(\mathbf{a}) 0^{\circ}$; (b) $26^{\circ}$; (c) $45^{\circ}$; (d) $64^{\circ}$; and (e) $90^{\circ}$. Atoms are colored according to their atomic heights.

\section{Conclusions}

In summary, we performed MD simulations to investigate the fundamental deformation mechanisms of nanotwinned Al containing inclined TBs under spherical nanoindentation. It was found that the plastic deformation of nanotwinned $\mathrm{Al}$ is dominated by individual deformation modes of dislocation slip, dislocation-TB interaction, and TB migration, the interaction between which is strongly dependent on the inclination angle of TBs with respect to the indented surface. The mean work hardening rate in the plastic regime increases with increasing inclination angle of TBs. Specifically, for an inclination angle of $45^{\circ}$, the critical force and displacement for the elastic-plastic transition are the highest, and the surface pile-up morphology is the most pronounced.

Author Contributions: Y.L., Y.D. and J.Z. conceived the project, Y.L. and J.Z. performed the simulations, Y.D. and J.Z. wrote the manuscript.

Funding: This research was funded by the National Natural Science Foundation of China (51875119 and 61473096), National Key R\&D Program of China (2016YFB0501203), the Fundamental Research Funds for the Central Universities, and the Science Challenge Project (No. TZ2018006-0201-02).

Conflicts of Interest: The authors declare no conflict of interest.

\section{References}

1. Pharr, G.M.; Oliver, W.C. Measurement of thin film mechanical properties using nanoindentation. MRS Bull. 1992, 17, 28-33. [CrossRef]

2. Saha, R.; Nix, W.D. Effects of the substrate on the determination of thin film mechanical properties by nanoindentation. Acta Mater. 2002, 50, 23-38. [CrossRef]

3. Lu, L.; Chen, X.; Huang, X.; Lu, K. Revealing the maximum strength in nanotwinned copper. Science 2009, 323, 607-610. [CrossRef] [PubMed]

4. $\quad$ Li, X.Y.; Wei, Y.J.; Lu, L.; Lu, K.; Gao, H.J. Dislocation nucleation governed softening and maximum strength in nano-twinned metals. Nature 2010, 464, 877-880. [CrossRef] [PubMed]

5. Pan, Q.S.; Zhou, H.F.; Lu, Q.H.; Gao, H.J.; Lu, L. History-independent cyclic response of nanotwinned metals. Nature 2017, 551, 214-217. [CrossRef] [PubMed]

6. Sun, L.G.; He, X.Q.; Lu, J. Nanotwinned and hierarchical nanotwinned metals: a review of experimental, computational and theoretical efforts. npj Comput. Mater. 2018, 4, 6. [CrossRef]

7. Bufford, D.; Liu, Y.; Wang, J.; Wang, H.; Zhang, X. In situ nanoindentation study on plasticity and work hardening in aluminium with incoherent twin boundaries. Nat. Commun. 2014, 5, 4864. [CrossRef] [PubMed]

8. Wei, Y.J. Anisotropic size effect in strength in coherent nanowires with tilted twins. Phys. Rev. B 2011, 84, 014107. [CrossRef]

9. Stukowski, A.; Albe, K.; Farkas, D. Nanotwinned fcc metals: Strengthening versus softening mechanisms. Phys. Rev. B 2010, 82, 224103. [CrossRef]

10. Brown, J.A.; Ghoniem, N.M. Reversible-irreversible plasticity transition in twinned copper nanopillars. Acta Mater. 2010, 28, 886-894. [CrossRef]

11. Zhang, J.J.; Hartmaier, A.; Wei, Y.J.; Yan, Y.D.; Sun, T. Mechanisms of anisotropic friction in nanotwinned Cu revealed by atomistic simulations. Model. Simul. Mater. Sci. Eng. 2013, 21, 065001. [CrossRef] 
12. Mishin, Y.; Farkas, D.; Mehl, M.J. Papaconstantopoulos, D.A. Interatomic potentials for monoatomic metals from experimental data and ab initio calculations. Phys. Rev. B 1999, 59, 3393. [CrossRef]

13. Rautioaho, R.H. An interatomic pair potential for aluminium calculation of stacking fault energy. Phys. Status Solidi B 1982, 112, 83-89. [CrossRef]

14. Kelchner, C.L.; Plimpton, S.J.; Hamilton, J.C. Dislocation nucleation and defect structure during surface indentation. Phys. Rev. B 1998, 58, 11085-11088. [CrossRef]

15. Honeycutt, J.D.; Andersen, H.C. Molecular dynamics study of melting and freezing of small Lennard-Jones clusters. J. Phys. Chem. 1987, 91, 4950-4963. [CrossRef]

16. Plimpton, S. Fast parallel algorithms for short-range molecular dynamics. J. Comput. Phys. 1995, 117, 1-19. [CrossRef]

17. Stukowski, A. Visualization and analysis of atomistic simulation data with OVITO-the Open Visualization Tool. Model. Simul. Mater. Sci. Eng. 2010, 18, 015012. [CrossRef]

18. He, Y.S.; Sun, T.; Yuan, Y.; Zhang, J.J.; Yan, Y.D. Molecular dynamics study of the nanoimprint process on bi-crystal Al thin films with twin boundaries. Microelectron. Eng. 2012, 95, 116-120. [CrossRef]

(C) 2018 by the authors. Licensee MDPI, Basel, Switzerland. This article is an open access article distributed under the terms and conditions of the Creative Commons Attribution (CC BY) license (http:/ / creativecommons.org/licenses/by/4.0/). 